and (b) "the science of physical (my italics) life, dealing with organised beings or animals and plants, their morphology, physiology, origin and distribution".

E. D. MYERS

Department of Postgraduate Medicine

University of Keele

Stoke-on-Trent ST4 7QB

\section{Journal Supplements}

SIR: I must register most strongly my objections to your recent Supplement Progress in Antidepressant Therapy (Journal, September 1988, 153). I was initially delighted that another supplement had arrived, especially with such a title-suggesting a review of recent trends in psychopharmacology. It was thus with dismay that I found that it was merely a prolonged advertisement for fluoxitene-presumably manufactured by Eli Lilly \& Co., as a quarter of the contributors worked for that company.

While I do not doubt that this new drug will prove to be an effective (and expensive) antidepressant, it seems inappropriate that the launch of a new product should be given such official sanction as its own supplement in the Journal-especially as it shares its properties with more established drugs already on the market, most notably fluvoxamine (but also clomipramine and trazodone).

If the Journal continues to publish the proceedings of symposia, which I trust it will, I hope they will report those meetings that are not so actively involved in the marketing of a particular product, but rather the collection together of informed professionals. I am sure that Eli Lilly \& Co. can afford to produce their own glossy advertising without the help of the Journal and thereby the tacit endorsement of the Royal College of Psychiatrists.

Matt Jelley

Barrow Hospital

Barrow Gurney

Bristol BS19 3SG

The allegation that the Journal s Supplement No. 3 is a "prolonged advertisement" for a particular product is untrue. This supplement is characterised, in fact, by carrying no advertising of any kind. It consists wholly of scientific information, much of it from outstanding scientific workers, which was peer-reviewed in accordance with the Journal's usual standards. The fact that a number of papers refer to a particular drug does not alter this situation. In the same way, the Archives of General Psychiatry recently published five papers in one issue and two in a subsequent issue all referring to a particular drug without, so far as I am aware, its integrity being challenged.
The publication of this supplement followed the requirements laid down by the College's Council, and conformed to the highest ethical standards; the content of all three supplements so far published has been fully endorsed by the Journal Committee. Of the Journal's 12000 subscribers (and considerably more readers), three have so far expressed disapproval of this particular supplement: none have been able to point to any fault in the content of any of the papers contained in it. If, as Dr Jelley suggests, the papers indicate that fluoxetine is the same in its properties as other drugs, that would be a rather peculiar form of 'advertising'.

This supplement does not in any way, directly or indirectly, offer "official sanction" or "tacit endorsement" to fluoxetine or any other drug; nor will any future issue do so. It is quite possible, however, that future supplements may contain more than one paper relating to a particular drug, provided that those papers are of appropriate scientific quality and conform to the Journal's normal requirements.

Editor, British Journal of Psychiatry

Hugh Freeman

\section{Prevalence of Dementia}

SIR: Ineichen (Journal, 1987, 150, 193-200) proposes that most of the reported prevalence rates of dementia in the elderly are too high, and in particular that the Newcastle rates are too high (Kay et al, 1964, 1970). However, the rate cited for the overall prevalence of dementia by Kay et al (1970) is shown as $8.8 \%$ (Table I of Dr Ineichen's paper), although the correct figure as given in the text is $6.2 \%$. This is almost exactly the median of the rates arranged in order of magnitude in this Table. The Newcastle rate of $6.2 \%$ for age $65+$ included cases which although mild (as opposed to severe) were regarded as definite.

Table II of Dr Ineichen's paper shows the rates reported for severe and severe + moderate dementia where authors make this distinction. The Newcastle rate is shown as $6.2 \%$ for severe dementia, but was actually $4.9 \%$ in the study cited (Kay et al, 1964). Moderate dementia was not distinguished but some cases which other authors might call moderate may have been rated as mild.

Dr Ineichen suggests that three recent UK studies which obtained low prevalence rates (mean $=2.9 \%$ ) are to be preferred to earlier studies. However, the data cited in his paper do not appear to support this view. Of the seven studies with rates of severe dementia below 3\% (Table II) (eleven others show rates $\geqslant 3 \%$ ) three refer only to people in their 70 th year, an 